\title{
Associative symmetry: IV. Classical conditioning in humans
}

\author{
LEONARD BROSGOLE and STUART J. LEFKOWITZ \\ St. John's University, Jamaica, New York 11439
}

\begin{abstract}
Two experiments were performed in which a subthreshold tone was paired with a light that followed. It was found that the subsequent presentation of the light alone was occasioned by the experience of a very low tone. We concluded that the light gained the capacity to generate a phenomenal tone by virtue of backward associations formed during training.
\end{abstract}

The question of associative symmetry derives from the classical conditioning literature and has been tested traditionally by use of backward conditioning procedures. Although backward conditioning has been demonstrated on an autonomic level (Champion \& Jones, 1961), for the most part, the behavioral evidence has not supported symmetry. Apparently, the implicit responses that may be conditioned in a backward fashion are either not expressed overtly or are manifested in a way that has not been scrutinized experimentally. We have come to adopt the latter position, which is to say that attempts at backward conditioning have focused upon inappropriate responses.

It is not surprising to find an animal salivating to a neutral stimulus (such as a tone) when that stimulus is followed by food. For, after all, under appropriate circumstances the tone will trigger the expectancy that the food will be presented at some time in the future. Accordingly, the conditioned response (CR) of salivation is based upon the mediating anticipation of events yet to come. However, the presentation of the neutral or conditioned stimulus (CS) in backward conditioning procedure is associated with and denotes the termination of the unconditioned stimulus (UCS). Since the CS is not utilized to signal or forecast future events in backward conditioning, it would seem ludicrous to focus upon the incremental development of the $\mathrm{CR}$ as evidence for associative symmetry. Rather, symmetry might be evidenced in the elicitation of such responses as scanning the field in search of the UCS, as in the case of food. The following studies represent an attempt to resolve the symmetry issue by using a backward conditioning procedure that was designed to produce a response quite appropriate to the peculiarities of the experimental setting.

\section{EXPERIMENT I}

The logic of this study was really very simple,

The authors wish to express their appreciation to Robert Zenhausern for his most helpful assistance throughout the course of this research. but hopefully not simple-minded. It merely involved presenting human observers with a sequence of events, a tone followed by a light, using a delay conditioning procedure. It was reasonable to expect that a relationship between the two stimuli would build up over trials, so that the tone would come to elicit the anticipation of the light. However, this study focused upon whether the light, when presented alone, would elicit the experience of tone on the basis of associative symmetry. Of course, it may seem totally absurd to expect that people would have heard a tone when it was conspicuously absent, but the absurdity wanes as the conditions employed made the absence of the tone quite inconspicuous. In fact, the intensity of the tone was adjusted for each subject so that it was $3 \mathrm{~dB}$ below the $50 \%$ limen.

\section{Method}

General. Although the logic of this study was simple, its execution became rather involved. The first step required obtaining the subject's threshold for the tone employed in the study. This was followed by the presentation of 200 trials, with the tone followed by the light half the time and the light alone on half the trials. The subject was required to respond as to whether or not the tone was heard by depressing pushbuttons. Two problems were recognized and corrected. First, the procedure was long and arduous. To maintain a level of alertness, it was necessary to precede the tone with an auditory warning signal. Second, it was feared that the threshold level might change as a function of being placed in a work situation. Therefore, threshold was obtained in the conditioning setting itself.

Subjects. Six males and six females participated in this study. Ranging in age from 19 to 28 years, their mean age was 22.4 years. All were naive as to the purpose of the experiment. Apparatus. Testing took place in a dimly lit Industrial Acoustics chamber (Model 1203A), with the subject seated at a table along a wall dividing the chamber from an adjoining control room. Communication between the chamber and control room was accomplished by use of a Fannon intercom system. The entire experimental sequence was controlled by a logic circuit composed of BRS digibit components (Series 200). The warning signal was produced by a BRS Sonalart unit (SA 201) which gave rise to a .1-sec chirp-like sound. The unit was located at the left rear corner of the table. The tone that followed was $900 \mathrm{~Hz}$. It was produced by a BRS audio oscillator (AO 201) and was fed through a BRS audio amplifier (AA 201) into a General Radio audio-frequency microvolter (Model 546C) which was used to regulate the intensity of 
the tone. The output of the microvolter was fed into a Lafayette stereophonic headset which was worn by the subject. A Masonite box, 7 in. wide $x 5$ in. deep, was located on the table directly in front of the subject. A $12-\mathrm{V}$ dc bulb was mounted centrally in a panel that extended upward vertically from the rear of the box. This light, which followed the tone, was powered by the BRS circuit. Two pushbutton switches were mounted to the horizontal surface of the box. They were 5 in. apart laterally, one to the left and the other to the right of the subject. The switches served as response keys, indicating the manner in which the subject experienced the tone. Switch closures were recorded on a BRS printout counter (POC-012) with a hand tally kept as a back-up system.

Procedure. The subjects were told that this was an experiment in subliminal perception and that they would be presented with tones of varying intensity-the loudest being barely audible. Sometimes there would be no tone at all. They were to prepare themselves to listen for the tone upon hearing an audible chirp. Sometime after the chirp, a light would come on and go off. Upon the light going off, they were to indicate whether the tone was heard by pressing one of the two buttons located on the box in front of them-the button to the left if the tone was heard before the onset of the light, and the one to the right if the tone came on with or after the light. They were instructed to respond if they heard, felt, or in any way sensed the presence of the tone. No response was required if the tone was not heard. These instructions were specifically designed to drop the criterion level in a rather conservative subject population. However, the subjects were thoroughly informed about the intent of the study at the conclusion of the procedure.

Threshold was obtained for the $900-\mathrm{Hz}$ tone prior to the conditioning trials using the method of limits. Fifteen ascending and 15 descending trials were counterbalanced. Each presentation of the tone was followed by the light, with the subject responding verbally through the intercom upon the offset of the light. Threshold was established as the mean of the last five ascending and five descending trials. (Threshold ranged from 42 to $45 \mathrm{~dB}$, re: $1_{\mathrm{uv}}$.) The tone was then adjusted $3 \mathrm{~dB}$ below that mean to compensate for any further drops in criterion or increases in sensitivity.

The threshold determination was followed by 200 trials. The light followed the tone in half the trials (Condition 1) and appeared alone in the remaining half (Condition 2). The order of the two conditions was counterbalanced according to the Fellows' series (1967). In the first condition, the tone followed the .1-sec ready signal by $1 \mathrm{sec}$ and was $2 \mathrm{sec}$ in duration. The light then appeared 1 sec after the onset of the tone and lasted for $2 \mathrm{sec}$. Thus, the tone and light overlapped for 1 full sec. The light followed the chirp by $2 \mathrm{sec}$ in Condition 2 , where the tone was omitted. Therefore, the timing sequence in the two treatments was identical. There was a 4-sec intertrial interval that commenced with the offset of the light. In essence, the procedure was a signal detection paradigm which allowed for the recording of hits, misses, correct rejections, and false alarms.

\section{Results and Discussion}

Each subject participated in a total of 200 trials, with the tone actually presented 100 times. Across subjects, then, there was an opportunity for 1200 hits (Condition 1) and 1200 false alarms (Condition 2). Accordingly, the data were broken down into hits and false alarms (Factor A), which was further divided into whether the experience of tone (be it a hit or a false alarm) occurred prior to the light or was produced by the light (Factor B). Because it was speculated that associative symmetry would be evidenced in terms of a progressive increase in false alarms as a function of trials, the hit and false alarm data were even further organized into five successive blocks of 20 trials each (Factor $\mathrm{C}$ ). The data were then subjected to a randomized block factorial design, using a standard transformation for frequency scores $(\sqrt{\mathrm{X}+.5})$.

There were a total of 1,070 hits in Condition 1, compared to 379 false alarms in Condition 2. This difference was significant $(F=69.58, \mathrm{df}=1 / 11$, $\mathrm{p}<.01$ ) which, of course, was no surprise.

Breaking the hit data down in five blocks of trials, there were 208, 211, 215, 221, and 215 hits from the first to the last block of 20 trials, respectively. In Condition 2 there were $52,76,78,84$, and 89 false alarms, respectively. There was a significant effect of trials upon the frequency with which the tone was reported $(F=3.52, \mathrm{df}=4 / 44, p<.05)$. Subsequent Newman-Keuls indicated that there was a significant increase in the tendency to report the tone from Block 1 to 2, with no further increment between adjacent blocks. Although the raw data seem to attribute the increased reporting of the tone to false alarms, trials did not interact significantly with hits and false alarms $(F=1.21, \mathrm{df}=4 / 44)$. Thus, we were left with the possible interpretation that the observed increase in false alarms may have been due to a mere dropping of criterion rather than the development of a bidirectional connection between the tone and light. However, there are several facts that argue against this interpretation.

For one, signal detection analysis revealed a significant decrease in sensitivity from the first to the second block of trials $(p<.05)$, with no further change thereafter. This would tend to indicate that the noted increase in the frequency of reporting the tone was due to a decrease in the discriminability between the two conditions which may have been based upon the formation of symmetrical connections.

Second, it was quite reasonable to expect an increase in hits. To begin with, the presentation of the light may have triggered the experience of tone on the basis of symmetry, thereby transforming a miss into a hit. In addition, it must be remembered that the tone was preceded by a warning signal which, also, may have transformed some misses into hits on the basis of forward conditioning. Thus, the hits should have consisted of correct detections plus actual false alarms generated by both the warning signal and light. Therefore, the subjects should have responded in terms of primarily hearing the tone before the onset of the light in Condition 1. Similarly, the warning signal may have produced some false alarms in Condition 2. In the main, however, they should have been generated by the light. Out data support this speculation. Of the 1,070 hits in Condition 1, there were 791 reports of the tone being heard before the onset of the light and 279 either 
with or after the appearance of the light. On the other hand, 95 false alarms occurred prior to the light in Condition 2, with 284 coincident with the onset of the light. Accordingly, there was a significant A by B interaction $(F=22.58, \mathrm{df}=1 / 11, \mathrm{p}<.01)$, indicating that the subjects were responding quite differently in the two conditions and not indiscriminately reporting the apparent presence of a tone. Simple effects showed that the tone was heard to precede the light significantly more often in Condition $1(\mathrm{~F}=14.11$, $\mathrm{df}=1 / 22, \mathrm{p}<$ .01 ), whereas just the opposite obtained in Condition 2 $(\mathrm{F}=23.33, \mathrm{df}=1 / 22, \mathrm{p}<.01)$.

Finally, the most convincing supporting evidence derived from phenomenological data obtained at the conclusion of each session. Each subject reported hearing two levels of tone. One was louder, more defined, and easily localized as coming from the earphones. The other seemed to emerge with the appearance of the light. It was much lower, fuzzy, and seemed to float freely overhead. Although the experience was very real, the subjects felt that they were often hallucinating. Some subjects found it difficult to terminate the tone. These reports were substantiated by the authors who participated in the study. Of course, their data are not included. Although the propensity of the light to produce a phenomenal tone was so striking, it was felt that a cleaner experiment was needed to document the contention of symmetry.

\section{EXPERIMENT II}

A serious argument can be raised against the previous study, namely, that the instructions and procedure were designed to effect a dramatic reduction in criterion, thereby sensitizing the subject to hear a tone upon the presentation of any stimulus. Such sensitization was evidenced by some subjects being unable to terminate the free-floating tonal experience. The following experiment attempted to control against the possibility of pseudoconditioning, as well as sensitization, by employing a discriminative conditioning paradigm.

\section{Method}

General. In accord with the logic of this experiment, two lights were used instead of one. They were separated horizontally so as to be quite discriminable. While the subthreshold tone was coupled with one of the lights (positive light), the other (negative light) followed the warning signal with no tone presented. The positive light was on the right for half the subjects and on the left for the remaining half. The procedure was divided into two halves. The first half consisted of conditioning trials where the tone and the positive light were presented on some trials and the negative light was shown on others. The tone was omitted during the second half of the procedure, thus enabling both lights to generate false alarms. It was reasoned that a certain number of false alarms should have been generated by the warning signal, as well as a heightened propensity to respond affirmatively. These should have been distributed equally between both lights. However, the formation of bidirectional connections during the first half of the procedure should have resulted in significantly more false alarms to the positive light, particularly at the time of its onset.

Subjects. Three males and five females participated in this study. Ranging in age from 18 to 22 years, their mean age was 19.0 years. All were naive as to the purpose of the experiment.

Apparatus. The previously used response box was modified by fixing two $12-\mathrm{V}$ dc bulbs to the rear panel. They were $24 \mathrm{in}$. apart horizontally from center to center. One was 12 in. to the left of the subject, and the other 12 in. to the right. The BRS circuitry was not altered in any way, so that the trials progressed just as before. Only the program was modified so as to include 10 additional trials. The purpose for this will be discussed below.

Procedure. The subjects were told that this was a probability learning experiment where a subthreshold tone would be differentially paired with two lights. They were informed that the probabilities would vary over subjects, so that one person might always receive the tone to one light and not at all to the other, while the tone might be distributed equally between the lights for another individual. They were further told to be keenly aware of the lights, so that they would be in a position to estimate the relative probabilities at the conclusion of the procedure. The subjects were instructed in the use of the response box, just as in the last experiment. The instructions were designed to maintain the discriminability of the lights, as pilot research indicated that subjects were closing their eyes and merely responding to flashes seen through the lids. Of course, the purpose of the study was fully explained upon the debriefing that followed the procedure. Threshold determinations were then taken, as in the prior study, and the tone was set $3 \mathrm{~dB}$ below threshold. The experimental procedure then commenced.

The first 100 trials were divided equally into presenting either the tone with the positive light or the negative light alone. The order of presentation was counterbalanced by use of the Fellows' series. This constituted a training session that was followed by 110 test trials.

The 110 test trials were divided into 10 blocks of 11 trials, with the positive light exposed six times in each block. However, the tone was presented only once with the positive light, on a random basis, in each block of trials. Thus, the positive light appeared alone five times per block, as did the negative light, allowing for a total of 50 false alarms to each. The tone was sporadically presented in this fashion in an attempt to preclude the recognition of its continued absence. Again, the Fellows' series was used in counterbalancing the order of exposing the positive and negative lights.

\section{Results and Discussion}

The data were broken down into false alarms to the positive and negative lights (Factor $\mathrm{A}$ ) and as to whether the false alarms occurred prior to or with the onset of the light (Factor B). In addition, the false alarms to the two lights were organized into five intervals of 10 trials each (Factor $\mathrm{C}$ ). Thus, we were able to assess the effect of trials upon the false alarms. These data were analyzed by use of a randomized block factorial design using the standard transformation previously described.

There were 400 false alarms possible to each stimulus across subjects. While 99 were committed to the positive, 47 were made to the negative light. This difference was significant $(\mathrm{F}=20.52, \mathrm{df}=1 / 7, \mathrm{p}<.01)$.

Of the 99 false alarms to the positive light, 23 preceded the light and 76 occurred with or after its onset. As to the 47 false alarms to the negative stimulus, 
26 preceded its onset, while 21 seemed to be produced by it. Accordingly, there was a significant A by B interaction $(F=18.90, \mathrm{df}=1 / 7, \mathrm{p}<.01)$. Simple effects revealed that significantly more false alarms occurred to the onset of the positive, compared to the negative stimulus $(F=38.77$, $\mathrm{df}=1 / 14, \mathrm{p}<.01)$. In addition, significantly more false alarms occurred with or after the appearance of the positive light than there were prior to onset $(F=41.45, \mathrm{df}=1 / 14, \mathrm{p}<.01)$. No other differences were significant.

From the first to the last block of 10 trials, there were $19,27,22,19$, and 17 false alarms to the positive stimulus, respectively. As to the negative stimulus, there were $5,5,11,9$, and 17 from the first to fifth block of trials, respectively. The analysis indicated a significant $A$ by $C$ interaction $(F=3.90$, df $=4 / 28$, $\mathrm{p}<.05$ ). (There was no significant main effect of $C$.) Simple effects showed that there were significantly more false alarms to the positive stimulus in the first $(F=7.04, \mathrm{df}=1 / 35, \mathrm{p}<.05)$, second $(F=18.02$, $\mathrm{df}=1 / 35, \mathrm{p}<.01)$, and third $(\mathrm{F}=5.26, \mathrm{df}=1 / 35$, $p<.05)$ block of trials. The differences thereafter were insignificant.

It would appear, then, that symmetrical connections were being formed between the tone and positive stimulus during the training sessions. These connections enabled the positive stimulus to generate the tonal experience, particularly at the time of onset. Furthermore, the difference in false alarms between the positive and negative stimuli diminished as the testing session progressed, indicating that the two stimuli were becoming less discriminable. This was to be expected because the testing session, by virtue of the removal of the tone, constituted a procedure for the extinction of the conditioned discrimination.

Again, our findings were supported by phenomenological evidence. The subjects reported that the tone became less intense and more vague with the passage of time, presumably during the testing phase of the procedure. In addition, while the tonal experience persisted along with the positive stimulus during the test, the negative light had the propensity to shut down the tone. It would go off almost immediately, even in those instances in which the apparent tone seemed to occasion the onset of the negative light. According to our subjects, then, the negative stimulus did not remain neutral. Rather, it seemed to become a conditioned inhibitor.

Accordingly, we may conclude that quantitative and phenomenological data strongly support the symmetry position with regard to classical conditioning.

\section{REFERENCES}

Champion, R., \& Jones, J. Forward, backward, and pseudoconditioning of the GSR. Journal of Experimental Psychology, $1961,62,58-61$.

Fellows, B. J. Change in stimulus sequences for discrimination tasks. Psychological Bulletin, 1967, 67, 87-92.

(Received for publication October 26, 1975.) 